Vous pouvez facilement suivre sur ces dessins, relatifs au développement de l'œuf d'hiver et à celui des œufs des formes agames, la première apparition de la masse génitale, puis son élongation, sa subdivision en une série de quatre petits corps disposés en chapelet. Alors que ces corps donnent naissance, dans les formes agames, à toute une série de chambres ovigères, dans la forme sexuée nous ne trouvons que deux de ces chambres, mais tout d'abord identiques; puis l'une de ces chambres s'atrophie, et l'autre, dont la prédominence s'accuse de plus en plus, finit par produire l'œuf unique, d'un volume si inusité.

Nous arrivons maintenant aux caractères absolument spéciaux à l'œuf d'hiver, à savoir : à sa fécondation et à la suspension dans les phénomènes du developpement de l'embryon proprement dit. Vous pouvez suivre sur ces figures la pénétration du spermatozoïde dans l'appendice polaire inférieur. Voici une petite masse dérivant du noyau primitif de l'œuf et paraissant pouvoir être assimilée à l'élément femelle primitif.

Ces deux masses voisines nous semblent représenter les deux corps mâle et femelle qui vont se fusionner.

Du résultat de cette fusion proviendrait cette masse globuleuse plurinucléaire qui, suivie dans son développement après la ponte, s'entourerait d'une sorte de coque relativement épaisse, véritable membrane kystique qui nous expliquerait la suspension dans ses phénomènes de développement. La présence de cette enveloppe accessoire si intéressante se traduit en coupe optique par une double ligne circulaire des plus accentuées.

\title{
Descriptions de Lépidoptères nocturnes
}

Par Paul THIERRY-MIEG.

1. Cidaria satanica, n. sp. - $\lesssim, 38$ mill. - Antennes noires, presque filiformes. Ailes supérieures d'un noir légèrement brunàtre, avec des bandes ondulées vert olive, comme suit : la base vert olive, limité par une mince ligne tremblée noire, ombrée de blanc extérieurement, qui part de la côte à 3 mill. de la base et va rejoindre le bord interne à 4 mill. de la base. Puis vient une bande verte, transversale, se terminant à la côte à 8 mill. de la base et à l'angle interne à cette même distance. Cette bande est très découpée extérieurement, limitée par un filet blanc, et sur le milieu de la bande on voit une ombre transversale noire. Le reste de l'aile est noir, avec une ligne tremblée 
blanche, partant de la côte à 4 mill. de l'apex et suivant le bord externe à 4 mill. Cette ligne devient verte et double à la hauteur de la première branche de la médiane, et vient se terminer au bord interne, à 4 mill. de l'angle interne. De l'endroit où cette ligne rencontre la $\Lambda^{\text {re }}$ branche de la médiane, part une bande irrégulière, verte bordée de blanc intérieurement, qui aboutit sur la côte à 10 mill. de l'apex. De là jusqu'à l'apex, la còte est mouchetée de vert. De l'apex part un petit trait droit, mais oblique, vert, long de 4 mill. On voit plusieurs taches vertes au bord externe. La frange est noire. Ailes inférieures noir uni, ainsi que la frange. Palpes noirs, verdâtres en dessous. Front jaune clair? Yeux noirs. Vertex et thorax d'un vert mousse. Dessus de l'abdomen noir. Dessous des ailes supérieures noir avec la base et le bord interne blanc grisâtre. Une petite tache grise à l'apex. Frange noire. Ailes inférieures blanches, avec un point cellulaire noir, et une bordure noire au bord externe, large de 5 mill. près de l'apex et beaucoup plus mince ( 2 mill.) a l'angle anal. Frange noire. Pattes noires annelées de jaune verdâtre. Dessous de l'abdomen jaunâtre.

Bolivie, 1 beau $\sigma^{\tau}$, coll. Staudinger.

2. Gidaria ignifera, n. sp. - $\sigma^{\star}, 36$ mill. - Antennes filiformes. Espèce voisine de $\boldsymbol{C}$. probataria $\mathrm{H}$.-S. Ailes supérieures très prolongées à l'apex, avec un angle vers le milieu du bord externe, d'un vert mousse avec 3 bandes transverses d'un vert plus foncé, la $1^{\text {re }}$ près de la base, la $2^{\text {e }}$ large de 6 mill. sur le milieu de l'aile, sur laquelle se voit un trait cellulaire allongé, noir, entouré de gris, la $3^{e}$ assez large, près de l'apex, et se rétrécissant avant d'arriver au bord interne. Elle est bordée de chaque côté d'une fine ligne blanche, interrompue. Le trait oblique, apical, commun à beaucoup de Cidaria, est représenté ici par une tache d'un gris brun, de 7 mill. de long, assez large dans sa deuxième partie, aboutissant à la ligne blanche qui borde intérieurement la $3^{\text {e }}$ bande transverse. Frange noirâtre, entrecoupée de vert. Ailes inférieures longues de $\mathbf{1 4}$ mill., d'un rouge brique, avec une bande grise, large de 6 mill., le long du bord abdominal; un faisceau de poils jaunâtres contenus dans une gouttière anale le long du bord abdominal, avant l'angle anal. Frange entrecoupée de rouge et de gris, et de quelques poils jaunes. Palpes gris, front jaunâtre (?), thorax vert, dessus de l'abdomen mélangé de vert, de rouge et de jaune. Dessous des ailes, comme chez $C$. probataria. Ailes supérieures noires avec la côte rougeâtre, avec une tache d'un blanc jaunâtre entre le trait cellulaire et l'apex, une autre à l'apex et une au milieu du bord externe. Une 
série de points internervuraux de mème couleur longe le bord externe à $\mathbf{2}$ mill. Frange noire, entrecoupée de jaune. Ailes inférieures d'un rouge brique pâle, avec plusieurs lignes ondulées, transverses, grises, la dernière, assez large, longe le bord externe. Frange entrecoupée de gris et de rougeâtre. Pattes noirâtres, annelées de gris, dessous de l'abdomen gris.

Bolivie, $1 \sigma^{\star}$, coll. Staudinger.

3. Cidaria Laodice, n. sp. - Taille et port de C. picata Hb. - $\overbrace{}^{\star}$, 30 mill. - Antennes pubescentes. Ailes supérieures, avec les mêmes dessins et courbes de lignes que chez $C$. picata. Le fond est vert mousse clair avec 3 bandes transverses d'un brun noirâtre, les 2 pre-

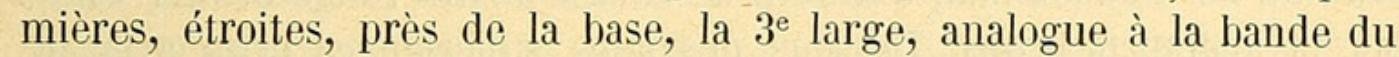
milieu de $\boldsymbol{C}$. picata, sur laquelle se détache un espace vert mousse allant de la còte à la jonction des $1^{\text {re }}$ et $2^{\text {e }}$ branches de la médiane. Un trait cellulaire noirâtre. Sur la còte, à 3 mill. de l'apex, se voit une tache d'un brun noirâtre, au-dessous de laquelle est un trait gris d'ardoise. Une ombre d'un brun noirâtre longe le bord externe, sauf en dessous de l'apex. Frange entrecoupée de brun et de vert. On voit par ce qui précède que les espaces, blancs chez $C$. picata, sont d'un vert mousse ici. Ailes inférieures d'un blanc jaunâtre luisant, avec un petit point cellulaire noir et la base grise. Frange blanche de l'apex au milieu du bord externe; de là à l'angle anal, elle est entrecoupée de noir. Front jaunâtre (à moins qu'il n'ait changé de couleur iu ramollissoir, ce qui arrive fréquemment pour les espèces vertes), thorax vert olive, dessus du corps noir. Dessous avec les lignes comme chez $C$. picata, mais aux ailes supérieures les parties grises sont noirâtres, la frange est presque entièrement noire, les ailes inférieures sont plus jaunes. Les 4 points cellulaires sont bien apparents. Pattes noires, annelées de jaunâtre.

Bolivie, $1 \delta^{\star}$, coll. Staudinger.

4. Cidaria Kirschi Maassen, var. Zalmoxis, n. var. - Absolument identique à $\boldsymbol{C}$. Kirschi, Maassen, pl. 9, f. 10, aux différences ci-après. Les dessins bruns du dessus des ailes supérieures sont remplacés par du vert mousse pâle, légèrement entrecoupé de brun. On voit sur le disque, à ร̆ mill. de l'apex, une tache d'un brun châtaigne dont le côté externe est noir. Aux ailes inférieures, le bord abdominal est moins noir que chez $\boldsymbol{C}$. Kirschi, et il n'y a pas de traces de noir à l'apex. Dessous comme chez $C$. Kirschi.

Bolivie, $1 \delta^{\star}$, coll. Staudinger. 
Il faudrait voir plusieurs exemplaires pour savoir si cette variété ne forme pas une espèce à part.

ร. Gidaria minois, n. sp. - $\sigma^{2}, 28$ mill. - Antennes filiformes. Ailes supérieures brunes, avec deux lignes transverses d'un brun très clair, presque droites, entre la base et le point cellulaire, qui est noir et très gros. De là au bord externe se voient 2 ou 3 lignes transverses, d'un brun très clair, ondulées, appuyées de plusieurs lignes plus foncées, également ondulées. Frange brune. Ailes inférieures d'un j̣aune clair, avec un petit point cellulaire brun et une bande brune un peu rétrécie dans son milieu longeant tout le bord externe. Un petit trait brun au bord abdominal, à 3 mill. de l'angle anal. Frange brune. Thorax et abdomen bruns. Dessous des ailes supérieures brun avec le point cellulaire comme en dessus, et une bande large de 2 mill. jaune clair, partant de la côte a $\breve{~ m i l l . ~ d e ~ l ' a p e x ~ e t ~ s e ~ t e r m i n a n t ~ s u r ~ l a ~}$ $3^{\text {e }}$ branche de la médiane. Une petite tache d'un jaune clair à l'apex. La partie de la côte qui avoisine l'apex, ainsi que le bord externe, sont d'un brun presque noir, ainsi que la frange. Ailes inférieures, comme en dessus, avec la frange brune un peu blanche à l'apex. Pattes grises annelées de jaunâtre.

Vallée de Cauca (Amérique du Sud) 1 ơ, ma coll.

6. Sabulodes mimula, n. sp. —đ̊, 3ə̈ mill. - Antennes filiformes. Dessus absolument identique comme forme et dessins à Sabulodes franciscata Dognin, mais le fond des ailes est d'un brun plus foncé. On voit 2 lignes transverses, d'un vert jaunâtre, ondulées, partant de la côte, la $1^{\text {re }}$ à 6 mill. de la base, la $2^{e}$ à 3 mill. de l'apex. Cette dernière, concave dans sa deuxième partie, aboutit au bord interne à 4 mill. de l'angle interne. Elle est appuyée intérieurement d'une ombre noirâtre, assez étendue. Une ligne ondulée d'un vert jaunâtre longe le bord externe des ailes inférieures à une distance de 4 mill. Un petit point cellulaire noir aux 4 ailes. Thorax et abdomen d'un brun foncé. Dessous très différent de $S$. franciscata, chez qui le jaunâtre domine. Ailes supérieures d'un brun noir uni, avec un trait cellulaire gris cendré et des traces de même couleur à l'apex. Ailes inférieures d'un blanc cendré, avec une bordure large de ร̈ mill. d'un brun noir, longeant le bord externe. Un petit point cellulaire noir. Frange brune aux 4 ailes, dessus et dessous. Dessous du thorax, avec des poils bruns, puis gris. Pattes et dessous de l'abdomen gris.

Bolivie, 1 ot, coll. Staudinger. 
7. Sabulodes mima, n. sp. - Le dessus rappelle un peu mon Sabulodes ornatissima. - o, 33 mill. - Antennes filiformes, brunes, picotées de blanc à la base. Les ailes supérieures ont un angle peu marqué au bord externe à la hauteur de la $1^{\text {re }}$ branche de la médiane et les ailes inférieures ont un rudiment de queue au milieu du bord externe. Ailes supérieures d'un brun noisette. La côte est d'un brun foncé picotée de blanc depuis la base sur une longueur de 7 mill. En dessous, on voit une bande d'un gris violet, appuyée elle-mème par une bande d'un gris verdâtre, qui part du bord interne, près de la base, vient d'abord aboutir obliquement un peu au-dessous de la naissance de la $3^{\mathrm{e}}$ branche de la médiane, puis, échancrée intérieurement, vient se terminer sur la nervure costale à 6 mill. de la base. On voit un espace brun orangé entre la naissance de la $3^{\mathrm{e}}$ branche de la médiane et le point de jonction des 2 premières branches de cette nervure. Une ligne de taches internervurales d'un gris verdâtre, arquées intérieurement, longe le bord externe à 3 mill. depuis l'apex (qui est picoté de blanc) jusqu’à la $2^{\mathrm{e}}$ branche de la médiane. Deux taches semblables se trouvent, mais a 5 mill. du bord externe, la première entre les $2^{\mathrm{e}}$ et $3^{\mathrm{e}}$ branches de la médiane, la $2^{\mathrm{e}}$ entre la $3^{\mathrm{e}}$ branche de la médiane et la sous-médiane. Ces deux taches sont ombrées de noir violâtre extérieurement. Enfin une dernière tache, d'un gris verdâtre, sous la sous-médiane, à 3 mill. de l'angle interne. Un petit point cellulaire blanc, frange brune. Ailes inférieures brun noisette, avec une ligne de taches internervurales gris verdâtre, arquées intérieurement, longeant le bord externe à 3 mill. L'espace entre cette ligne et le bord externe est plus foncé que le reste des ailes. Un très petit point cellulaire blanc. Frange brune. On voit enfin une ligne, commune aux quatre ailes, d'un brun foncé, qui, partant de la côte des ailes supérieures à environ 6 mill. de l'apex, traverse ces ailes en zigzag, et passant sur le point cellulaire des ailes inférieures, vient aboutir au bord abdominal à 4 mill. de l'angle anal. Front, vertex et épaulettes d'un brun foncé, pérygodes d'un gris violet picoté, dessus de l'abdomen brun. Dessous des ailes supérieures blanc depuis la base jusqu'à la moitié de l'aile. Le reste d'un brun noir, sauf l'apex qui est blanc. Frange d'un brun noir, blanche à l'apex. Ailes inférieures blanches, avec une bordure d'un brun noir longeant le bord externe. Cette bordure a 3 mill. de large à l'apex et s'amincit vers la moitié de son parcours, pour se terminer très étroitement à l'angle anal. Frange d'un brun noir. Dessous du thorax brun très clair près de la tète, puis blanc, pattes antérieures brunes en dessus, blanches en dessous, ailes 
inférieures blanches un peu renflées, dessous de l'abdomen d'un blanc jaunâtre.

Pérou, 1 ta ma coll ; 1 t, coll. Staudinger.

8. Erateina luceria, n. sp. - Coupe d'ailes à peu près comme E. undulata Saund. - ot, 24 mill. - Antennes noires, filiformes. Ailes supérieures noires, avec une large bande blanche transparente, partant de la costale, à 1 mill. de la còte, et à 7 mill. de la base, large d'abord de 2 mill. et finissant sur la $3^{\text {e }}$ branche de la médiane (où elle est large de 3 mill.), à 3 mill. du bord externe. Frange noire entrecoupée de blanc. Ailes inférietires noires, très étroites ( 6 mill.), longues de 16 mill. y compris une petite prolongation caudale; une petite tache rouge au bord abdominal près de l'angle anal, surmontée, dans l'intérieur de l'aile, de 2 petits traits blancs, formant ligne; frange noire, entrecoupée de blanc. Thorax et corps noirs, ce dernier annelé de jaunâtre. Dessous des ailes supérieures d'un rouge cinabre, avec la bande diaphane du dessus atteignant la còte, un trait cellulaire blanchâtre et une ligne jaune d'or, irrégulièrement ondulée, partant de la côte à 3 mill. de l'apex et venant finir à l'angle anal. Frange comme en dessus. Ailes inférieures d'un rouge cinabre, avec un large repli de mème couleur, couvert de lignes blanches, allant de la base jusqu'à 7 mill. du bord abdominal. Après ce repli, les nervures sont marquées de blanc et on voit une ligne blanche partant de la côte à 3 mill. de l'apex et finissant par un crochet au bord abdominal à 3 mill. du repli. Cette ligne blanche est appuyée extérieurement par une bande jaune d'or partant de l'apex et finissant au bord abdominal. Franges comme en dessus. Palpes noirs, blancs en dessous, pattes noires annelées de blanc, abdomen blanc.

Bolivie, 2 ơ $^{\star}$, ma coll.

9. Scordylia quadruplicaria Hb., var. latior, n. var. - Le type de S. quadruplicaria Hb., 603, 604, nous donne la bande jaune transverse et oblique du milieu des ailes supérieures mince et formée de 3 taches, les 2 premières, formant une bande, sont généralement réunies, la dernière est isolée et se trouve près du bord interne. La même disposition se retrouve en dessous.

Je propose de donner le nom de var. latior aux exemplaires qui ont ces 3 taches réunies en une seule bande, large de 2 mill. se terminant très près de l'angle interne. La bande est également complète en dessous.

Pérou, $2 \delta^{*}$; Santa-Catharina, $1 c^{*}$, ma coll. 
10. Scordylia quadruplicaria Hb., var. latissima, n. var. - Cette variété est l'exagération de la précédente. La bande a 2 mill. à la còte, mais elle s'élargit immédiatement et atteint 4 mill. de large. Elle se termine également tout près de l'angle interne. Les deux bandes des ailes inférieures sont un peu plus larges que chez le type dans la var. latior, et encore davantage dans la var. latissima.

Pérou, 2 ơ, ma coll.; 1 s, collection Saudinger.

11. Clysia Gaujoni, n. sp. - $ð, 47$ mill. - Antennes filiformes; très voisine de Clysia columbipennis Wk., mais les quatre ailes sont d'un gris plus foncé. L'angle du bord externe des ailes supérieures est bien moins prononcé. On voit une ligne oblique d'un gris brun, un peu flexueuse, partant de la còte à 9 mill. de la base et rejoignant le bord interne à 10 mill. de la base. Une deuxième ligne, concave, part de la côte à 3 mill. de l'apex et vient aboutir au bord interne à 3 mill. de l'angle interne. On voit une tache de rouille sur la deuxième branche de la médiane et une autre, plus petite, sur la troisième branche de la médiane. Ces deux taches sont appuyées extérieurement contre la deuxième ligne mentionnée plus haut. Ailes inférieures avec une ligne droite d'un gris brun partant de la cote à ว̆ mill. de l'apex et rejoignant le bord abdominal à 4 mill. de l'angle anal. Un petit point cellulaire noir aux quatre ailes. Dessous un peu plus clair que le dessus (mais plus obscur que chez $C$. columbipennis), avec une bande d'un gris brun, commune aux quatres ailes, longeant le bord externe sans l'atteindre, sauf aux ailes supérieures entre l'indépendante et la $\mathbf{1}^{\text {re }}$ branche de la médiane. Les pattes ne sont pas tachetées de brun, mais d'un gris foncé presque uni.

Environs de Loja (Équateur), 6 ơ, ma coll. et coll. Dognin.

Dédiée à M. l'abbé Gaujon. Je tiens cette espèce de la générosité de M. P. Dognin.

12. Azelina exquisita, n. sp. - $\sigma^{\star}, 46$ mill. - Antennes grises, crénelées. La côte des ailes supérieures est fortement bombée un peu avant l'apex. Les ailes sont sans dentelures. Ailes supérieures d'un gris brun, avec la base d'un gris cendré et des rayures plus claires sur la côte. Cet espace gris cendré est limité par une ligne noire, partant de la côte à 8 mill. de la base. Cette ligne est d'abord largement convexe jusqu'à la naissance de la $3^{\text {e }}$ branche de la médiane, puis elle vient atteindre-obliquement, en formant d'abord une petite convexité, le bord interne, à $\check{~ m i l l . ~ d e ~ l a ~ b a s e . ~ U n e ~ l i g n e ~ b r u n e, ~ p r e s q u e ~ d r o i t e, ~}$ part de la côte à 10 mill. de l'apex, et aboutit au milieu du bord 
interne. Enfin une ligne, d'abord d'un blanc rosé (pendant 1 mill.), puis cendrée, bordée de noir des deux còtés, part de la còte à 7 mill. de l'apex et, concave vers la fin, vient finir au bord interne, a 6 mill. de l'angle interne. Le reste des ailes, entre cette ligne et le bord externe, est d'un gris luisant, marbré à certaines places de noir et de brun. Quelques points noirs internervuraux, parfois mouchetés de blanc, se trouvent au bord externe. La côte est d'un blanc rosé rayé de gris rosé depuis 8 mill. de la base sur un parcours de 9 mill. Frange d'un gris brun. Ailes inférieures d'un gris brun; la moitié des ailes, depuis la base, est rayée d'une foule de lignes droites, brunes. Le reste des ailes est finement strié de brun noir, et l'on voit 3 ou 4 points internervuraux, noirs, au bord externe, près de l'angle anal. Frange grise. Palpes et front gris, thorax gris cendré, abdomen gris brun. Dessous des ailes supérieures gris rosé, finement strié d'une multitude de petites lignes noires, transverses. Une ligne blanche, transverse, presque droite, part de la côte à š mill. de l'apex, et vient aboutir au bord interne, à こ̆ mill, de l'angle interne; mais elle est alors presque effacée. Toute la partie des ailes située entre cette ligne et le bord externe est noire. La côte est grise, sans stries noires depuis la base jusqu’à ə̌ mill. de l'apex. Un trait cellulaire blanc, allongé. Frange noire. Ailes inférieures d'un rouge brique pâle, nuancé par places de gris rosé, avec une foule de stries transverses jaunâtres, un trait cellulaire blanc, et une ligne ondulée, blanche, partant de la còte à š mill. de l'apex et venant finir au bord abdominal, a ら mill. de l'angle anal. Ces ailes sont picotées de-ci de-là de petites écailles noires. Frange brune. Pattes brunes picotées de jaunâtre, dessous de l'abdomen d'un rouge brique très pâle, presque gris.

Pérou, 1 ऽ̊, ma coll.

\section{Du sexe chez les Seydmaenidae}

\section{Par J. CROISSANDEAU.}

La détermination du sexe chez les Scydmænidæx est extrêmement difficile. Les premiers monographes ont établi leurs doctrines sans les approfondir, le plus souvent par analogie, ou sur quelques exemplaires isolés.

En ce qui regarde les Cephennium, M. Reitter avait cru voir tout d'abord, dans la protubérance basale, un caractère mâle; mais il semble 


\section{$2 \mathrm{BHL}$ Biodiversity Heritage Library}

Thierry-Mieg, Paul. 1894. "Descriptions de Lépidoptères nocturnes." Annales de la Société entomologique de France 63, 51-58.

View This Item Online: https://www.biodiversitylibrary.org/item/101302

Permalink: https://www.biodiversitylibrary.org/partpdf/238517

\section{Holding Institution}

Harvard University, Museum of Comparative Zoology, Ernst Mayr Library

\section{Sponsored by}

Harvard University, Museum of Comparative Zoology, Ernst Mayr Library

\section{Copyright \& Reuse}

Copyright Status: Public domain. The BHL considers that this work is no longer under copyright protection.

This document was created from content at the Biodiversity Heritage Library, the world's largest open access digital library for biodiversity literature and archives. Visit BHL at https://www.biodiversitylibrary.org. 